\title{
Gene co-expression modulating terpene metabolism is associated with plant anti-herbivore defence during initial flowering stages
}

\author{
Zheng Ling ${ }^{1}$, Jingrui $\mathrm{Li}^{1}$, Yan Dong ${ }^{1}$, Wen Zhang ${ }^{1}$, Hongtong Bai ${ }^{1}, \mathrm{Shu} \mathrm{Li}^{2}, \mathrm{Su} \mathrm{Wang}^{2}$, Hui \\ $\mathrm{Li}^{1}$, and Lei Shi ${ }^{1}$ \\ ${ }^{1}$ Institute of Botany Chinese Academy of Sciences \\ ${ }^{2}$ Beijing Academy of Agricultural and Forestry Sciences
}

May 5, 2020

\begin{abstract}
To protect themselves from herbivore attacks, especially during early developmental stages, plants produce volatiles possessing various ecological and physiological functions. Here, we identified three genes cloned from Lavandula angustifolia. These genes, designated as LaTPS7, LaTPS8, and LaCYP71D582, were hypothesized to be active in plant defense during early developmental stages. The in-vitro assays showed LaTPS7 produced eight compounds including camphene, myrcene, limonene etc. and LaTPS8 catalyzed nine volatiles from $\alpha$-pinene, sylvestrene to fenchol etc. using geranyl diphosphate (GPP) and nerolidyl diphosphate (NPP) as substrate separately. However, LaTPS7 present in plastids only, synthesized limonene within Nicotiana benthamiana. Limonene was then converted into carveol by LaCYP71D582 present in the endoplasmic reticulum. LaTPS8, also located in plastids, synthesized $\alpha$-pinene and sylvestrene. Odour response of aphids (Myzus persicae) and ladybugs (Harmonia axyridis) showed that volatiles from transgenic tobacco leaves repelled aphids and attracted ladybugs. LaTPS7 promoter GUS stain assay in Arabidopsis thaliana showed that LaTPS7 displayed a wound-induced expression in leaves. Together, our findings show that these compounds and gene-expression pattern played important roles in protecting plants during vulnerable stages. More practically, these plant tactics can be exploited in agriculture to decrease the use of insecticides, thereby contributing to improved human and environmental health.
\end{abstract}

Gene co-expression modulating terpene metabolism is associated with plant anti-herbivore defence during initial flowering stagesZhengyi Ling ${ }^{1,2}$, Jingrui $\mathrm{Li}^{1}$, Yanmei Dong ${ }^{1}$, Wenying Zhang ${ }^{1}$, Hongtong Bai ${ }^{1}$, Shu Li ${ }^{3}$, Su Wang ${ }^{3}$, Hui Li ${ }^{1 *}$, Lei Shi ${ }^{*}{ }^{*}$

${ }^{1}$ Key Laboratory of Plant Resources and Beijing Botanical Garden, Institute of Botany, Chinese Academy of Sciences, Beijing 100093, China

${ }^{2}$ University of Chinese Academy of Sciences, Beijing 100049, China

${ }^{3}$ Institute of Plant and Environment Protection, Beijing Academy of Agricultural and Forestry Science, Beijing 100097, China

*Corresponding authors.

\section{Corresponding author:}

Prof. Lei Shi

Institute of Botany, Chinese Academy of Sciences

Xiangshan, Beijing 100093, China

Telephone: 86-10-62836270 
E-mail:

shilei_67@126.com

Dr. Hui Li

Institute of Botany, Chinese Academy of Sciences

Xiangshan, Beijing 100093, China

Telephone: 86-10-62836054

E-mail:lihui@ibcas.ac.cn

\section{Gene ID:}

BankIt2292418 LaTPS7 MN822897

BankIt2292495 LaTPS8 MN822898

BankIt2292498 LaCYP71D582 MN822899

\section{Abstract}

To protect themselves from herbivore attacks, especially during early developmental stages, plants produce volatiles possessing various ecological and physiological functions. Here, we identified three genes cloned from Lavandula angustifolia. These genes, designated asLaTPS7, LaTPS8, and LaCYP71D582, were hypothesized to be active in plant defense during early developmental stages. Thein-vitro assays showed $L a$ TPS7 produced eight compounds including camphene, myrcene, limonene etc. and La TPS8 catalyzed nine volatiles from $a$-pinene, sylvestrene to fenchol etc. using geranyl diphosphate (GPP) and nerolidyl diphosphate (NPP) as substrate separately. However, La TPS7 present in plastids only, synthesized limonene withinNicotiana benthamiana. Limonene was then converted into carveol by La CYP71D582 present in the endoplasmic reticulum. La TPS8, also located in plastids, synthesized $a$-pinene and sylvestrene. Odour response of aphids (Myzus persicae) and ladybugs (Harmonia axyridis) showed that volatiles from transgenic tobacco leaves repelled aphids and attracted ladybugs. LaTPS7 promoter GUS stain assay inArabidopsis thaliana showed that LaTPS7 displayed a wound-induced expression in leaves. Together, our findings show that these compounds and gene-expression pattern played important roles in protecting plants during vulnerable stages. More practically, these plant tactics can be exploited in agriculture to decrease the use of insecticides, thereby contributing to improved human and environmental health.

Key words : lavender, monoterpenes, terpene synthase, cytochrome P450, insects plant-herbivore-natural enemy tritrophic interaction

\section{Introduction}

Plants interact with the environment by releasing various volatiles that possess physiological and ecological functions (O'Connor, 2015). The theory of herbivore-induced plant volatiles (HIPVs), proposed by Ehrlich and Raven (1964), states that plants release various volatiles to communicate and defend against herbivore attack. Thereafter, studies have been increasingly focused on relationships between plant compounds and insects. A recent report showed that the cottons emitting increased levels of volatile compounds have fewer eggs of Helicoverpa armigera (Liu et al. 2018), suggesting that volatiles play important roles in protecting plants from pests and enhancing plant survival. Indeed, numerous plant-derived volatile compounds, particularly terpenes and terpenoids, are documented to protect plants. For example, farnesene protects plants from aphids (Myzus persicae ) because it acts as an alarm pheromone and attracts the predatory ladybugs (Harmonia axyridis ) (Francis et al.2004; Zhu et al. 2005). Another common compound in flowers and fruits is linalool, which has studied in Arabidopsis thaliana, indicating it attracts thrips; however, oxides of linalool, catalysed by CYP71D, can repel these insects (Boachon et al . 2015). Meanwhile, HIPVs, including mint-derived volatiles such as limonene, 1,8-cineole, and carvone, can also attract species that predate on herbivores, such as predatory mites that feed on two-spotted spider mites (Togashi et al. 2019). Therefore, 
volatile compounds may be part of a defence system used directly or indirectly by plants to protect themselves via tritrophic interactions (Meena et al . 2017). Furthermore, as they deter herbivores, these plant volatiles are likely expressed in higher concentrations during early stages of development than in mature stages and tissues (Meena et al. 2017); thereafter, expression of these compounds is maintained at a constant level or reduced( Niederbacher et al . 2015). This shows that plants dynamically regulate expression of metabolites in order to adapt to the environment and reproduce.

Most HIPVs are terpenes/terpenoids and are biosynthesized via two pathways. The first is the mevalonate (MVA) pathway, which occurs in the cytosol/endoplasmic reticulum, and leads to the formation of farnesyl diphosphate (FPP) as a precursor to sesquiterpenoids. The other is 2-C-methyl-D-erythritol-4-phosphate (MEP) pathway, occurring in plastids; this pathway generates geranyl diphosphate (GPP) as a precursor to monoterpenoids, catalysed by the associated terpene synthases (TPSs) (McGarvey and Croteau, 1995). These TPSs are a mid-sized gene family in plants, consisting of TPS-a, -b, -c, -d, -e/f, and -g (Chen et al . 2011).

Recently, neryl diphosphate (NPP), which is an isomer of GPP, and (Z,Z)-FPP, were shown to be precursors to several terpenes in the tomato plant (Schilmilleret al. 2009; Akhtar et al . 2013). This indicates that terpene/terpenoid biosynthesis needs to be further investigated in various plant species. Moreover, the structure and function of terpenes that are post-processed by co-expressed genes have been extensively studied in the peppermint (Mentha x piperita) (Rodneyet al . 2005), Artemisia annua L. (Teoh et al . 2006), and A. thaliana (Boachon et al , 2019). However, few studies have examined these compounds in the lavender.

Lavender (Lavandula angustifolia) is an important aromatic plant generating as many as 70 volatile metabolites, including limonene, $a$ - pinene, linalool. Lavender has also been proposed as a model to study the regulation of terpene biosynthesis (Guitton et al.2010a). Although lavender metabolism has been investigated for several years, enzymes, such as 3 -carene synthase, fenchol synthase, $a$-pinene synthase, and $\beta$-phellandrene synthase are mainly identified usingin-vitro assays (Demissie ZAet al . 2011; Benabdelkaderet al . 2014; Adal AM et al . 2017). In addition, terpenes synthesized by TPSs are potential to be transported into endoplasmic reticulum where these compounds are converted by cytochrome P450 (CYP) into their respective derivatives (Karunanithi and Zerbe 2019). This enables plants to increase the expression of metabolites that can perform multiple functions. Although the CYP enzyme family, which accounts for $1 \%$ of the genome in most plants (Nelson and Werck-Reichhart 2011), has not been functionally identified in the lavender, 30 unique CYPs genes were predicted using sequence tags (ESTs) (Lane et al. 2010).

Physiological functions of volatiles and their genes within tritrophic interactions in lavenders remain elusive. Previous studies have described volatile organic compound (VOC) content during inflorescence ontogeny, and revealed that the terpenes involved fell into three groups identifiable via three developmental phases of flowering. 3-carene, limonene, myrcene, bornyl acetate, borneol, camphor, 1,8-cineol, and trans -ocimene belonged to the first group and may play a protective role in repelling damaging insects (Guittonet al . 2010b). Previously, we found that aphids and ladybugs were dominant insects in lavender fields during early spring in Beijing. This suggests probable tritrophic interaction among predators, preys and volatiles (Li et al.2019) . Y-tube olfactometer experiments showed $\beta$-trans-ocimene and (+)-R-limonene get rid of $74.71 \%$ and $78.41 \%$ aphids (Li et al. 2019).

In this study, we investigated the tactics used by the lavender plant to defend against herbivore attack during immature developmental stages and in young plant tissues.

\section{MATERIALS AND METHODS}

\section{Plants and insects}

Lavender (L. angustifolia) plants were harvested at different developmental stages from a field belonging to the Institute of Botany, Chinese Academy of Sciences, China. The three distinct stages were as follows: B, bud stage: the petals were completely closed and green; I, blossom stage: the $60 \%$ of flowers had opened and 
petals turned purple; II, fade stage: the petals had shrunk and were ready to fall. Four-week-old tobacco $(N$ icotiana benthamiana ) and $A$. thaliana were grown in a green-house at $25^{\circ} \mathrm{C}$ under a $16 / 8 \mathrm{~h}$ light and dark photoperiod $\left(200 \mu \mathrm{mol} \mathrm{m} \mathrm{s}^{-2}\right)$.

Aphids (Myzus persicae) and ladybugs (Harmonia axyridis) were kindly provided by Prof. Su Wang (Beijing Academy of Agricultural and Forestry Sciences) and maintained in a growth chamber $(30 \times 30 \times 25$ $\mathrm{cm})$ at $25^{\circ} \mathrm{C}$ with a L16:D8 photoperiod feeding on tobacco plant.

\section{Clone and quantification expression analysis of LaTPS7,LaTPS8 and LaCYP71D582}

Glandular trichomes are tissues located in metabolism-specific sites (Tissieret al . 2017). Total RNAs were extracted from glandular trichomes of flowers and leaves in different developmental stages and reverse transcribed using oligo $\mathrm{d}(\mathrm{T})$ primers following the manufacturer's directions (TSINGKE, China). After obtaining cDNA, target genes were amplified using cloning primer and Phanta Max Polymerase (Vazyme, China). To clone full-length open reading frames (ORFs), we used a PCR program at the following settings: $95^{\circ} \mathrm{C}$ for $5 \mathrm{~min}$; 35 cycles at $95^{\circ} \mathrm{C}$ for $2 \mathrm{~min} ; 58^{\circ} \mathrm{C}$ for $30 \mathrm{~s}$ and $72^{\circ} \mathrm{C}$ for $2 \mathrm{~min}$; and final extension at $72^{\circ} \mathrm{C}$ for 5 min. The qRT-PCR was conducted using $2 \times$ T5 Fast qPCR Mix (SYBR Green II) (TSINGKE, China) on an Mx3000P system (Agilent Stratagene), according to the manufacturer's instructions and the following settings: $95^{\circ} \mathrm{C}$ for $1 \mathrm{~min}$, followed by 40 cycles of $10 \mathrm{~s}$ at $95^{\circ} \mathrm{C}$, then $5 \mathrm{~s}$ at $55^{\circ} \mathrm{C}$, and $15 \mathrm{~s}$ at $72^{\circ} \mathrm{C}$. Leaf trichomes at the blossoming stage were used as controls. Normalised expression values $\left(2^{-\mathrm{T}}\right)$ were calculated according to the expression of $18 \mathrm{~S}$ rRNA used as reference gene. The primers used in this part of our study are shown in table S1.

\section{Sequence analysis of target genes}

The full amino acid sequences translated from ORFs (primers are provided in table S1) were used for multiple sequence alignent performing on an ESPript (http://espript.ibcp.fr/ESPript/ESPript/) as described previously (Robert et al. 2014). Phylogenetic trees were constructed by MEGA7 using the Neighbor-Joining method, and Interactive Tree of Life (i TOL) was used for visual editing (http://itol2.embl.de/) (Letunicet al. 2007). Analysis of signal peptides in TPSs, and prediction localization of candidate proteins were performed on Phyre2 (http://www.sbg.bio.ic.ac.uk/phyre2) and signal P (http://www.cbs.dtu.dk/services/SignalP$4.1 /$ ) as described previously (Kelley et al.2015). Protein secondary structure was predicted using I-TASSER (http://zhanglab.ccmb.med.umich.edu/I-TASSER).

\section{Determination oftissue-specific gene expression in A. thaliana}

Approximately $1.5 \mathrm{~kb}$ of a promoter for each gene was isolated by FPNI-PCR from genomic DNA using three specific gene primers and three arbitrary primers and PCR settings described by Wang et al. (2011). Resulting sequences were analysed on PlantCARE (bioinformatics.psb.ugent.be/webtools/plantcare/html) (Rombauts et al. 1999) and then integrated into pCXGUS-P vector harbouring a cloning cassette upstream of GUS(uidA). After the constructs were confirmed via sequencing, they were introduced into GV3101Agrobacterium, which was used to transform A. thaliana using a floral dip method described previously (Clough et al. 1998). Transformed lines were selected on MS plates containing $50 \mu \mathrm{g} \cdot \mathrm{mL}^{-1}$ hygromycin, and were then confirmed via PCR. Enzymatic assay using 5-bromo-4-chloro-3-indolyl- $\beta$-D-glucuronide (GUS) was performed according to the method of Jefferson et al. (1987) to assess candidate genes tissue-specific expression.

\section{Subcellular localization of the LaTPS7, LaTPS8, and LaCYP71D582 in N. benthamiana}

GV3101 with eGFP expression vectors controlled by a mannopine synthase promoter (MAS), constructed using a Trelief SoSoo Cloning Kit (TSINGKE, China), were used for transient transformation of 4-weekold $N$. benthamiana plant as described by Jin et al. (2015). After 3 days of infiltration, $N$. benthamiana leaves were excised, mounted on slides, and analyzed using a confocal laser-scanning microscope equipped with a standard filter set (Leica TCS SP5). Images were processed by Image J (https://imagej.nih.gov/ij).

Heterologous expression of TPSs in Escherichia coli and enzymatic assayin vitro 
TPS sequences of truncated predicted signal peptide were inserted into a pDE2 vector with a recombinant C-terminal and poly-histidine tag using primers shown in table S1 and a pDE2 Directional Expression Kit Ver.2 (TSINGKE, China). The final constructs was transformed into E. coli BL21 (DE3); recombinant proteins were subjected to isopropyl- $\beta$-D-thiogalactopyranoside (IPTG) induction at the concentration of $1.0 \mathrm{mM}$ until $\mathrm{OD}_{600}$ reached $0.6^{\sim} 0.8$, and then to purification using a His-Tagged Gravity Column (Merck Millipore). The purified proteins were assayed by sodium dodecyl sulphate-polyacrylamide gel electrophoresis (SDS-PAGE) to ensure that the proteins had been extracted successfully.

The in-vitro enzymatic assay for TPS activity was performed according to the method of Chen et al. (2011) in a final volume of $500 \mu \mathrm{L}$ buffer [25mM HEPES, $\mathrm{pH} 7.3 ; 10 \mathrm{mM} \mathrm{MgCl} 2 ; 10 \mathrm{mM} \mathrm{MgCl}_{2} ; 10 \%$ glycerol; 10 mM DTT], approximately $20 \mu \mathrm{L}$ pure protein, and $10 \mu \mathrm{g}$ of either FPP, NPP, or GPP (Sigma-Aldrich). The mixtures were vortexed, incubated at $30^{\circ} \mathrm{C}$ for $2 \mathrm{~h}$, after which $250 \mu \mathrm{L}$ hexane was added to each mixture and vortexed for $1 \mathrm{~min}$. The upper layers were centrifuged at $1200 \mathrm{~g}$ and $4^{\circ} \mathrm{C}$ for $30 \mathrm{~min}$, and then transferred into 2-mL glass vials for analysis via GC-MS. Heat-inactivated recombinant protein was used as negative control.

\section{Transient expression of LaTPS7, LaTPS8, andLaCYP71D582 in N. benthamiana}

Leaves of $N$. benthamiana collected from 4-week-old plants were infiltrated with Agrobacterium tumefaciens GV3101 harboring target genes. Meanwhile, GV3101 was mixed with TPS and CYP at respective ratios of 1:1 when $\mathrm{OD}_{600}$ of each strain corresponded to 1.0. Leaf tissues were collected after 5 days of infiltration, examined under UV light to ensure successful agroinfiltration and gene expression, and then ground in liquid nitrogen. Leaf powder was stored at $-80^{\circ} \mathrm{C}$, and volatiles were extracted by vortexing in $70 \%$ alcohol $(1: 4 \mathrm{~m} / \mathrm{v})$. Extracts were maintained at $-20^{\circ} \mathrm{C}$ and oscillated before analysis via solid-phase microextraction (SPME, Agilent). The samples were heated in a water bath at $40^{\circ} \mathrm{C}$ for $30 \mathrm{~min}$, and then a fibre coated with polydimethylsiloxane and divinylbenzene was used to collect volatiles under the same conditions for $30 \mathrm{~min}$ according to a previously published method (Ilc et al. 2017). Volatiles were then analysed using GC-MS.

\section{GC-MS Analysis}

GC-MS analysis was performed via splitless injection using an Agilent 7890A GC system and Agilent Technologies 5975C Inert XL Mass Selective Detector equipped with an HP-5MS UI column $(30 \mathrm{~m} \times 0.25 \mathrm{~mm} \times$ $0.25 \mu \mathrm{m}$; Agilent Technologies). Injector temperature was $250^{\circ} \mathrm{C}$. Temperature was initially held for $3 \mathrm{~min}$ at $40^{\circ} \mathrm{C}$, then increased to $130^{\circ} \mathrm{C}$ at the rate of $10^{\circ} \mathrm{C} \cdot \mathrm{min}^{-1}$, increased to $250^{\circ} \mathrm{C}$ at the rate of $50^{\circ} \mathrm{C} / \mathrm{min}$, and maintained for $10 \mathrm{~min}$; helium gas at a flow of $1 \mathrm{~mL} / \mathrm{min}$ was used as vector.

MS conditions were as follows: ionization energy, $70 \mathrm{eV}$; electronic impact ion source temperature, $200^{\circ} \mathrm{C}$; quadrupole temperature, $150^{\circ} \mathrm{C}$; and mass range, $35-550 \mathrm{u}$. Products were identified based on their retention time and electron ionization mass spectra using mass spectra from the National Institute of Standards and Technology (NIST) Mass Spectral Library (NIST-14.0).

\section{Insect behaviour}

For aphids (Myzus persicae), a leaf disk bioassay was performed as described by Picard et al . (2012). Transformed $N$. benthamiana leaves were punched into disks of $1 \mathrm{~cm}$ in diameter. These disks were placed onto filter paper that was suitable for a Petri dish. Aphids, which had been starved for $3 \mathrm{~h}$, were placed in these Petri dishes, and the number of insects on either control disks or treatment disks was recorded for 1 hour. Ninety aphids were tested in total. We then used a Y-Tube olfactometer to assess the influence of volatiles on ladybugs (Harmonia axyridis ) that had been starved for $3 \mathrm{~h}$. Sixty ladybugs were tested in total. Ladybugs entering into more than half-length of the arms of the olphactometer and lingering for at least $10 \mathrm{~s}$ were recorded as responders. Disks were exchanged for fresh disks after 10 aphids or ladybugs were tested.

\section{Statistical analysis}

The statistics of aphids was assessed by variance (ANOVA). Means were separated using Tukey's HSD test 
at a significance level $p=0.05$. As for ladybugs (Harmonia axyridis), the statistics was assessed by using $\chi$ 2 test.

\section{RESULTS}

\section{TPS and CYP clustered in phylogenetic tree}

Two TPS genes having the sizes of 1803 and 1800 bp were obtained from the same primer, and were named LaTPS7 and LaTPS8, respectively; LaTPS7 and LaTPS8 displayed an identical nucleotide sequence in the first $33 \mathrm{bp}$ of $\mathrm{N}^{\prime}$ and in the last $23 \mathrm{bp}$ of C' (Figure S2). Phylogenetic trees based on deduced amino-acid sequences of each gene showed that these two genes belonged to the TPS-b subfamily, which consists of monoterpene synthases (Chen et al. 2011) (Figure 1a).LaTPS7showed the highest identity withL. angustifolia limonene synthase when searched via BLAST in NCBI, andLaTPS8 showed highest identity with $L$. peduncuiatapinene synthase in a phylogenetic tree. Both of these proteins contained the conserved arginine-tryptophan $\left(\mathrm{RRX}_{8} \mathrm{~W}\right)$ motif in the $\mathrm{N}$ terminal, and an aspartate-rich (DDXXD) and (NSE/DTE) motif (which chelates divalent metal ions such as $\mathrm{Mg}^{2+}$ or $\mathrm{Mn}^{2+}$ ) in the $\mathrm{C}$ terminal. Synthases need these motifs to cyclise terpene precursors such as GPP, NPP, or/and FPP (Figure S3, S4).

The CYP sequence was submitted to CYP450 nomenclature committee and designated as LaCYP71D582 . The protein showing highest identity with $L a$ CYP71D582 was $P$. barbatus $P b$ CYP71D378 (Figure 1b), which functions in forskolin (Pateraki et al. 2017) with conserved motifs (A/G)GX(D/E)T(T/S), EXXR, and FXXGXRXCXG (Figure S5). Secondary protein structure with four membrane-spanning domains and active site is shown in supplementary figure S5. CYP clans, used to build the phylogenetic tree, were related to terpene metabolism (Nelson and Werck-Reichhart, 2011), and the candidate CYP was clustered into the CYP71 clan, which is the largest clan among the CYPs (Figure 1b). This result indicates that it is highly possible to catalyze terpenes (Nelson et al. 2004). These results indicate that La CYP71D582 may participate in terpene metabolism

\section{Quantitative RT-PCR analysis of candidate-gene expression during budding stage}

The expression level of LaTPS7, LaTPS8 andLaCYP71D582 were accessed via quantitative real-time PCR (qRT-PCR) during different periods of budding, blossoming and fading stages of glandular trichomes in leaves and flowers (Figure 2). LaTPS7, LaTPS8, andLaCYP71D582 were highly expressed in the glandular trichomes of flower during budding stage, but showed low expression in other organs. Meanwhile, the expression levels of LaTPS7, LaTPS8 , andLaCYP71D582 decreased during blossoming and fading stage, showing a similar expression pattern, and indicating that the three genes perform similar functions during plant development (Figure 2). This expression pattern may provide a temporal basis for consecutive catalysis: products from TPSs may be further catalyzed by CYP.

Subcellular localization of the LaTPS7, LaTPS8, and LaCYP71D582 via transient expression in tobacco

When $L a$ TPS7 and $L a$ TPS8 were predicted by Signal P, the signal peptide was not detected in the plastid. However, the signal was detected when we used Phyre2, and $L a$ CYP71D582 was predicted to localize on the membranes by both Signal P and Phyre2. Hence, experimental verification is particularly important when prediction of TPSs subcellular localization is conflicted. Thus, full-ORF fusion vectors enhanced with green fluorescent protein (eGFP) were constructed for each candidate gene, and injected into N. benthaiana leaves via $A$. tumefaciens -mediated transfection. Analysis of whole leaves via confocal microscopy showed that both $L a$ TPS7 and $L a$ TPS8 were associated with chloroplasts (Figure 3), whereas $L a$ CYP71D582 was located in the endoplasmic reticulum (ER) (Figure 3).

\section{Isolation andin-vitro enzymatic characteristic of TPSs}

To determine the function of each protein, six His-tagged TPS recombinant proteins, with truncated putative signal peptides in their amino acid sequences, were expressed and extracted from E. coli BL21 (DE3). These recombinant proteins were then tested with either GPP, NPP, or FPP (10 $\mu$ g for each) used as 
substrate. Our results show that La TPS7 catalysed GPP to produce seven monoterpenes corresponding to $a$-pinene, camphene, myrcene, limonene, terpinolene, linalool, and terpineol, and catalysed NPP to $a$ -pinene, camphene, limonene, terpinolene, terpineol, and nerolidol. La TPS8 produced $a$-pinene, $\beta$-pinene, sylvestrene, linalool, fenchol, and geraniol from GPP, and $a$-pinene, limonene, terpinolene, terpineol, and nerolidol from NPP. However, no products were detected when FPP was used as substrate for both genes (Figure 4). These results indicate that these enzymes differ in their substrate recognition and product output.

\section{Functional characterization of TPSs and CYP in vivo}

To further identify the products of target proteins in plants,LaTPS7and LaTPS8 were separately transiently transfected into tobacco leaves. Similarly, LaCYP71D582 was co-expressed with either LaTPS7 or LaTPS8 to investigate the function of $L a C Y P 71 D 582$. Our results showed that $L a$ TPS7 produced only limonene within tobacco, whereas $L a$ TPS8 produced $a$ - pinene and sylvestrene; these results are consistent with those of the in-vitro enzymatic assay (Figure 5a). Notably, carveol levels were assessed in tobacco plants co-expressing LaTPS7 and LaCYP71D582, but no changes in caveol levels were observed in plants co-expressing LaTPS8 - LaCYP71D582 (Figure 5b). In other words, limonene could be hydroxylated at C6 by La CYP71D582 to form carveol (Figure 5c).

\section{Promoter clone and tissue-specific expression by GUS strain in $A$. thaliana}

Because LaCYP71D582 and LaTPS7 showed a relationship of successive catalysation, we further investigated the precise expression pattern of these genes. Thus, a 1299-bp promoter of LaTPS7 and a 1434-bp promoter of LaCYP71D582 were obtained via FPNI-PCR and designated asPro -LaTPS7and Pro -LaCYP71D582 . Both of these promoters not only contained the basic promoter elements, but also harboured several regulatory elements predicted by PlantCARE (Figure S6, S7). We then constructed $A$. thaliana lines transformed with each gene promoter-GUS fusion vectors. Strain assay ofLaTPS7showed that it was expressed in flowers, siliques, trichomes and leaves. Its expression in leaves, in particular, exhibited a wound-induced pattern (Figure 6a). In other words, LaTPS7 was only highly expressed in leaves after a leaf had been injured, similar to the response observed in leaves being attacked by herbivores. LaCYP71D582 showed a constitutive expression pattern in flowers, siliques, trichomes, and leaves (Figure $6 \mathrm{~b}$ ). This response was similar to that of LaTPS7, but expression of LaCYPr1D582 did not show a wound-induced pattern.

\section{Products of LaTPS7 and LaCYP71D582 protect plants against insects}

To the best our knowledge, limonene repels most herbivores including aphids, as shown in our previous work (Li et al. 2019). However, how limonene and its derivatives affect interactions between aphids and ladybugs remains undetermined. Therefore, to determine how limonene and its derivatives affect the relationship between aphids and ladybugs, we used a leaf disk assay and a Y-Tube olfactometer to assess responses from aphids and ladybugs, respectively, to tobacco leaves transiently transfected withLaTPS7and co-transfected withLaTPS7and LaCYP71D582 .

Our result show that tobacco leaves transfected with $L a$ TPS7, and leaves co-transfected with $L a$ TPS7 and $L a$ CYP71D582, repelled aphids at a rate of approximately $70 \%$ (Figure $7 \mathrm{~b}$ ). In contrast, La TPS7expressing tobacco leaves attracted ladybugs at a rate of $48.33 \%$, whereas leaves co-expressing $L a$ TPS7 and $L a$ CYP71D582 attracted ladybugs at a rate of $58.33 \%$ (Figure 7a). These results suggest that limonene and carveol were more attractive to ladybugs than limonene alone.

\section{Discussion}

Plants emit more volatiles during early stages of development than during mature stages because in order to grow and proliferate successfully, they must protect juvenile tissues, such as young leaves and buds, against herbivore attack (Meenaetalet al . 2017). This is consistent with our heat map of flower development (Figure S1), showing that various volatile compounds play significant roles in plant defence during early stages. Identification of gene function in the Japanese pepper (Zanthoxylum piperitum ) (Fujita et al. 2017) and in A. thaliana(Boachonet al. 2019) also showed that genes participating in defence are mainly active during early stages of development and in young tissues of plants. This is a time period when plants need to 
supply energy for growth and defensive strategies concurrently, which is difficult because each of these two pathways requires high energy expenditure (Meena et al et al . 2017). Collectively, these results indicate that genes and their products in the lavender bud stages likely function in plant defence.

Volatile compounds protect plants not only by repelling herbivores, but also by attracting the natural enemies of herbivores, forming a tritrophic interaction among plant-herbivores-carnivores. This provides means of natural biological control and may serve as a new approach to pest eradication, decreasing the use of insecticides, which are used excessively in modern agriculture and other industrial applications. Our results show that limonene and carveol, expressed in tobacco leaves, repel aphids and attract ladybugs. This has also been shown in soybeans (Glycine max ) and mint (ZHU et al . 2005; Togashi et al. 2019). Additionally, limonene attracts predatory mites, Phytoseiulus persimilis and Neoseiulus californicus(Togashi et al. 2019) and repels western flower thrips (Frankliniella occidentalis ) (Picardet al . 2012), indicating that limonene can defend a wide variety of plants (Smith et al . 2018). Combining carveol, pinene, and limonene is important in developing plant-derived insect repellents (Picard et al. 2012; Smithet al. 2018). However, other studies indicate that limonene promotes herbivore and pathogenic attack in the orange fruit (Rodriguezet al . 2011). Moreover, Musca domestica L. catalyses limonene into carveol and carvone via endogenous P450, resulting in lower toxicity to flies (Rossiet al . 2013). This suggests that the same terpene may function differently among different species; this needs to be investigated in future studies.

Terpenes or terpenoids in plants are mainly catalysed by terpene synthases (TPSs). The sequences and functions of TPSs are redundant, causing difficulty in annotation and assignment even in Gossypiumgenomes (Huang et al . 2018). The similarity betweenLaTPS7 and LaTPS8 is quite high, showing $77.61 \%$ identity; correspondingly, the products of these genes are highly redundant in vitro.However, both of LaTPS7 and LaTPS8 can catalyze GPP and NPP into various products, which shows the promiscuous and multi-substrate characteristics of TPSs (Major, 2012). Although most monoterpene synthases, including $L a$ TPS7 and $L a$ TPS8, are located in the plastids, exceptions exist and can be located in the mitochondria and cytosol ( Yamaga et al . 1993; Aharoni et al . 2004; Lee \& Chappell, 2008; Magnardet al. 2015). This suggests that there is a complex metabolic network of terpenes. Additionally, $L a$ TPS7 and $L a$ TPS 8 show different localization patterns in plastids. La TPS7 wrapped around chloroplasts, while La TPS8 distribution was punctate. Although reasons for these differences remain undetermined, these different patterns of localization may stem from different substrates in organelles. Moreover, several noncanonical metabolic pathways have been recently identified. For example, a novel enzyme called $R h$ NUDX1 (a nudix hydrolase) producing geraniol has been discovered in roses and constitutes a catalysis model that differs from known monoterpene synthases (Magnard et al . 2019). This indicates that our understanding of net-regulatory metabolism of terpenes, including substrate mediation and stimuli by environmental factors, are presently incomplete.

We used two promoters to examine regulation of terpene synthesis. Using the LaTPS7 promoter predicted by PlantCARE resulted in our discovery of MYC transcription factors (TFs), which helped explain the wound-associated pattern of expression observed via the GUS stain bioassay. An analogous pattern was discovered in Capsicum annuumL. (Wu et al. 2019; Wang et al. 2016). Studies on relationships between TFs and TPSs, such as those among NAC, ethylene-insensitive3-like TF, and R2R3-MYB, can be conducted in lavenders (Nieuwenhuizen et al.2015; Reddy et al. 2017). Although some of TFs have been demonstrated in L. $\times$ intermedia (Sarker et al . 2019), other regulatory factors modulating terpene release will likely be discovered in lavenders.

Conversely, CYPs participate in defence of a wide variety of plants. Plant CYPS include CYP51 in oats (Avena spp.), CYP99A2 and CYP99A3 in rice (Oryza sativa), and CYP82G1 in Arabidopsis(Qi et al . 2006; Shimura et al . 2007; Lee et al . 2010). LaCYP71D582, the first CYP gene cloned from lavenders, protects the plants by converting limonene into carveol to enrich terpenoid metabolites in early stages of flowering. Although some reports show that CYPs target to plastidial membranes (Kim et al.2010; Quinlan et al. 2012), we found that $L a$ CYP71D582 was localized to the endoplasmic reticulum (ER) (Figure 3), close to plastids within plant cells. This enabled putative communication between these two organelles, which was also shown by Ginglinger et al.(2013). LaCYP71D582 shared 57.68 and 7.98\% similarity with limonene-6- 
hydroxylase ( $M$. canadensis ) (Accession number: QDF63370.1) and limonene-6-hydroxylase $(M$. x gracilis ) (Accession number: AAQ18706.1), respectively, indicating that determining the function of a CYP only by its sequence may be unreliable.

The LaCYP71D582 promoter possesses elements for response to environmental stimuli, such as the GT1motif responsible for responding to light conditions, and the LTR sequence responsible for responding to low temperature. This indicates that CYPs are modulated by environmental factors. Unfortunately, studies on how environmental factors influence CYPs and terpenoid regulation are rare. CYPs participate in various catalytic and metabolic activities in plants, including metabolism of fatty acids and alkane compounds mediated by CYP72, 701, and 90, flavonoid metabolism mediated by CYP73, 98, and 84, and phytoalexin metabolism mediated by CYP79, 80, and 93 (Nelson and Werck-Reichhart, 2011). Therefore, it is important to study not only CYP-mediated catalysis but also biotic and environmental factors that influence CYP activity.

In summary, herein we examined the functions of three genes in lavenders. These genes were associated with direct and indirect plant protection during the budding stage. We also examined the interaction among the plants, aphids, and ladybugs. This tritrophic interaction is vital for the successful propagation of lavenders (Figure 8). Collectively, our results show that metabolism in lavenders during the budding stage involves different regulatory mechanism compared with that occurring during the blossoming stage. Our results also indicate that plants used multiple tactics to protect themselves against herbivores and offer a new perspective on biological pest control.

\section{ACKNOWLEDGMENTS}

We would like to thank Prof. Yongzhen Pang from Institute of Animal Science of Chinese Academy of Agricultural Sciences for her useful suggestions and language editing, which has greatly improved the manuscript. We thank Dr. Yan Zhu from Institute of Botany, the Chinese Academy of Sciences for her support in mass spectrum analysis.

\section{CONFLICT OF INTEREST}

The authors declare no conflict of interest.

\section{Authors'}

\section{contributions}

Z.L., H.L., and L.S. designed the study; Z.L. performed all experiments; J.L. performed bioinformatic analysis; Y.D., W.Z., and H.B. analyzed the chemical data; S.W., and S. L. performed insect rearing. Z.L., H.L., and L.S. analyzed the data and wrote the manuscript. All of the authors read and approved the final manuscript.

\section{Funding}

This work was supported by the Strategic Priority Research Program of the Chinese Academy of Sciences (Grant No. XDA23080603) and National Natural Science Foundation of China (Grant No. 31701956 ). The funder has no role in the design of the study and collection, analysis, and interpretation of data, or preparation of the manuscript.

\section{REFERENCES}

Akhtar T.A., Matsuba Y., Schauvinhold I., Yu G., Lees H.A., Klein S.E. \& Pichersky, E. (2013) The tomato cis-prenyltransferase gene family. The Plant journal: for cell and molecular biology 73, 640-52.

Adal A.M., Sarker L.S., Lemke A.D. \& Mahmoud S.S. (2017) Isolation and functional characterization of a methyl jasmonate-responsive 3-carene synthase fromLavandulax intermedia . Plant Molecular Biology 93 , 641-657. 
Aharoni A., Giri A.P., Verstappen F.W., Bertea C.M., Sevenier R., Sun Z. \& Bouwmeester H.J. (2004) Gain and loss of fruit flavor compounds produced by wild and cultivated strawberry species. Plant Cell16 , 3110-31.

Benabdelkader T., Guitton Y., Pasquier B., Magnard J.L., Jullien F., Kameli A. \& Legendre L. (2015) Functional characterization of terpene synthases and chemotypic variation in three lavender species of section Stoechas. Physiologia plantarum 153, 43-57.

Boachon B., Junker R.R., Miesch L., Bassard J.E., Höfer R., Caillieaudeaux R., ... Werck-Reichhart D. (2015) CYP76C1 (Cytochrome P450)-Mediated linalool metabolism and the formation of volatile and soluble linalool oxides in Arabidopsis Flowers: a strategy for defense against floral Antagonists. Plant Cell 27 , 297290.

Chen F., Tholl D., Bohlmann, J. \& Pichersky E. (2011) The family of terpene synthases in plants: a mid-size family of genes for specialized metabolism that is highly diversified throughout the kingdom. The Plant Journal $66,212-29$.

Clough S.J. \& Bent A.F. (1998) Floral dip: a simplified method for agrobacterium-mediated transformation of Ara bidopsis thaliana. The Plant Journal $16,735-43$.

Demissie Z.A., Sarker L.S. \& Mahmoud S.S. (2011) Cloning and functional characterization of $\beta$-phellandrene synthase from Lavandula angustifolia . Planta 233, 685-96.

Ehrlich P.R. \& Raven P.H. (1964) Butterflies and plants: a study in coevolution. Evolution 18 , 586-608.

Francis F., Lognay G. \& Haubruge E. (2004) Olfactory responses to aphid and host plant volatile releases: (E)-beta-farnesene an effective kairomone for the predator Adalia bipunctata. Journal of Chemical Ecology 30, 741-755.

Fujita Y., Koeduka T., Aida M., Suzuki H., Iijima Y. \& Matsui K. (2017) Biosynthesis of volatile terpenes that accumulate in the secretory cavities of young leaves of Japanese pepper (Zanthoxylum piperitum ): Isolation and functional characterization of monoterpene and sesquiterpene synthase genes. Plant Biotechnology $\mathbf{3 4}$, $17-28$.

Ginglinger J.F., Boachon B., Höfer R. \& Paetz C. (2013) Gene coexpression analysis reveals complex metabolism of the monoterpene alcohol linalool in Arabidopsis flowers. Plant Cell25 , 4640-57.

Guitton Y., Nicolè F., Moja S., Benabdelkader T., Valot N., Legrand S., .. Legendre L. (2010a) Lavender inflorescence: a model to study regulation of terpenes synthesis. Plant Signaling and Behavior $\mathbf{5}$, 749-51.

Guitton Y., Nicolè F., Moja S., Valot N., Legrand S., Jullien F. \& Legendre L. (2010b) Differential accumulation of volatile terpene and terpene synthase mRNAs during lavender (Lavandula angustifoliaand L. $\mathrm{x}$ intermedia ) inflorescence development.Physiologia plantarum 138, 150-63.

Huang X.Z., Xiao Y.T., Köllner T.G., Jing W.X. Kou J.F., Chen JY., .. Guo Y.Y. (2018) The terpene synthase gene family in Gossypium hirsutum harbors a linalool synthase Gh TPS12 implicated in direct defence responses against herbivores. Plant, Cell and Environment 41, 261-274.

Ilc T., Halter D., Miesch L., Lauvoisard F., Kriegshauser L., Ilg A., .. Navrot N. (2017) A grapevine cytochrome P450 generates the precursor of wine lactone, a key odorant in wine. The New phytologist 213 , 264-274.

Jefferson R.A., Kavanagh T.A. \& Bevan M.W. (1987) GUS fusions: beta-glucuronidase as a sensitive and versatile gene fusion marker in higher plants. The EMBO journal 20 , 3901-7.

Jin J., Kim M.J., Dhandapani S., Tjhang J.G., Yin J.L., Wong L., \& Jang I.C. (2015) The floral transcriptome of ylang ylang (Cananga odorata var. fruticosa) uncovers biosynthetic pathways for volatile organic compounds and a multifunctional and novel sesquiterpene synthase. Journal of experimental botany 66 , 3959-75. 
Karunanithi P.S. \& Zerbe P. (2019) Terpene synthases as metabolic gatekeepers in the evolution of Plant terpenoid chemical diversity.Frontiers in Plant Science 10 , 1166.

Kelley L.A., Mezulis S., Yates C.M., Wass M.N. \& Sternberg M.J. (2015) The phyre2 web portal for protein modeling, prediction and analysis. Nature Protocols10 , 845-858.

Kim J-E., Cheng KM., Craft NE., Hamberger B. \& Douglas CJ. (2010) Over-expression of Arabidopsis thaliana carotenoid hydroxylases individually and in combination with a $\beta$-carotene ketolase provides insight into in vivo functions. Phytochemistry71, 168-178.

Landmann C., Fink B., Festner M., Dregus M., Engel KH. \& Schwab W. (2012) Cloning and functional characterization of three terpene synthases from lavender (Lavandula angustifolia). Archives of Biochemistry and Biophysics 465, 417-29.

Lee S. \& Chappell J. (2008) Biochemical and genomic characterization of terpene synthases in Magnolia grandiflora. Plant Physiology 147, 1017-33.

Lee S., Badieyan S., Bevan D.R., Herde M., Gatz C. \& Tholl D. (2010) Herbivore-induced and floral homoterpene volatiles are biosynthesized by a single P450 enzyme (CYP82G1) in Arabidopsis . Proceedings of the National Academy of Sciences of the United States of America107 , 21205-21210.

Leroy P.D., Sabri A., Heuskin S., Thonart P., Lognay G., Verheggen FJ., ... Haubruge E. (2011) Microorganisms from aphid honeydew attract and enhance the efficacy of natural enemies. Nature Communications $\mathbf{2}, 348$.

Letunic I. \& Bork P. (2007) Interactive Tree of Life (i TOL ): an online tool for phylogenetic tree display and annotation.Bioinformatics $23,127-8$.

Li H., Li J.R., Dong Y., Hao H.P., Ling Z.Y., Bai H.T., ... Shi L. (2019) Time-series transcriptome provides insight into the gene regulation network involve in the volatile terpenoid metabolism during the flower development of lavender.BMC Plant Biology 19, 313.

Liu D., Huang X., Jing W., An X., Zhang Q., Zhang H., ... Guo Y. (2018) Identification and functional analysis of two P450 enzymes of Gossypium hirsutuminvolved in DMNT and TMTT biosynthesis. Plant Biotechnology Journal 16, 581-590.

Magnard J.L., Roccia A., Caissard JC., Vergne P., Sun P., Hecquet R., .. Baudino S. (2015) Plant volatiles. Biosynthesis of monoterpene scent compounds in roses.Science 349, 81-3.

Major D.T. \& Weitman M. (2012) Electrostatically guided dynamics - the root of fidelity in a promiscuous terpene synthase? Journal of the American Chemical Society 134, 19454-62.

McGarvey D.J. \& Croteau R. (1995) Terpenoid metabolism. The Plant Cell 7 , 1015-1026.

Meena R.K., Jangra S., Wadhwa Z., Monika. \& Wati L. (2017) Role of plant volatiles in defense and communication. International Journal of Current Microbiology and Applied Sciences . 6 , 300-313.

Nelson D. \& Werck-Reichhart D. (2011) A P450-centric view of plant evolution. The Plant journal : For Cell and Molecular Biology66 , 194-211.

Nelson D.R., Schuler M.A., Paquette S.M., Werck-Reichhart D. \& Bak S. (2004) Comparative genomics of rice and Arabidopsis. Analysis of 727 cytochrome P450 genes and pseudogenes from a monocot and a dicot. Plant Physiology 135, 756-72.

Niederbacher B., Winkler J.B. \& Schnitzler J.P. (2015) Volatile organic compounds as non-invasive markers for plant phenotyping. Journal of Experimental Botany . 66 , 5403-5416.

Nieuwenhuizen N.J., Chen X.Y., Wang M.Y., Matich A.J., Perez R.L., Allan A.C., .. Atkinson R.G. (2015) Natural variation in monoterpene synthesis in kiwifruit: transcriptional regulation of terpene synthases by NAC and ETHYLENE-INSENSITIVE3-like transcription factors. Plant Physiology 167, 1243-1258. 
O'Connor S.E. (2015) Engineering of Secondary Metabolism. Annual Review of Genetics 49 , 1-94.

Pateraki I., Andersen-Ranberg J., Jensen N.B., Wubshet S.G., Heskes A.M., Forman V., .. Hamberger B. (2017) Total biosynthesis of the cyclic AMP booster forskolin from Coleus forskohlii . eLife 6 , e23001.

Picard I., Hollingsworth R.G., Salmieri S. \& Lacroix M. (2012) Repellency of essential oils to Frankliniella occidentalis (Thysanoptera: Thripidae) as affected by type of oil and polymer release. Plant molecular biology . 93, 641-657.

Pichersky E. \& Raguso R.A. (2018) Why do plants produce so many terpenoid compounds. The New Phytologist 220, 692-702.

Qi X., Bakht S., Qin B., Leggett M., Hemmings A., Mellon F., .. Osbourn A. (2006) A different function for a member of an ancient and highly conserved cytochrome P450 family: From essential sterols to plant defense.Proceedings of the National Academy of Sciences of the United States of America103 , 18848-18853.

Quinlan R.F., Shumskaya M., Bradbury L.M.T., Beltrán J., Ma C., Kennelly E.J. \& Wurtzel E.T. (2012) Synergistic interactions between carotene ring hydroxylases drive lutein formation in plant carotenoid biosynthesis.Plant Physiology 160 , 204-214

Reddy V.A., Wang Q., Dhar N., Kumar N., Venkatesh .PN., Rajan C., .. Sarojam R. (2017) Spearmint R2R3-MYB transcription factor $M s$ MYB negatively regulates monoterpene production and suppresses the expression of geranyl diphosphate synthase large subunit (Ms GPPS.LSU). Plant Biotechnology Journal15 , 1105-1119.

Robert X. \& Gouet P. (2014) Deciphering key features in protein structures with the new ENDscript server. Nucleic Acids Research 2 , W320-W324.

Rodríguez A., San Andrés V., Cervera M., Redondo A., Alquézar B., Shimada T., .. Peña L. (2019) Terpene down-regulation in orange reveals the role of fruit aromas in mediating interactions with insect herbivores and pathogens. Plant Physiology, 156 , 793-802.

Rombauts S., Déhais P., Van Montagu M. \& Rouzé P. (1999) PlantCARE, a plant cis-acting regulatory element database. Nucleic Acids Research 27 , 295-6..

Rossi Y.E. \& Palacios S.M. (2013) Fumigant toxicity of Citrus sinensis essential oil on Musca domestica L. adults in the absence and presence of a P450 inhibitor. Acta Tropica127, 33-37.

Sarker L.S., Adal A.M. \& Mahmoud S.S. (2019) diverse transcription factors control monoterpene synthase expression in lavender (lavandula ). Planta 251, 5.

Schilmiller A.L., Schauvinhold I., Larson Me., Charbonneau A.L., Schmidt A., Wilkerson C., ... Pichersky E. (2009) Monoterpenes in the glandular trichomes of tomato are synthesized from a neryl diphosphate precursor rather than geranyl diphosphate.Proceedings of the National Academy of Sciences of the United States of America106 , 10865-70.

Shimura K., Okada A., Okada K., Jikumaru Y., Ko K.W., Toyomasu T., .. Yamane H. (2007) Identification of a biosynthetic gene cluster in rice for momilactones. The Journal of Biological Chemistry 282 , 34013-34018.

Smith G.H., Roberts J.M. \& Pope T.W. (2018) Terpene based biopesticides as potential alternatives to synthetic insecticides for control of aphid pests on protected ornamentals. Crop Protection 110 , 125-130.

Tissier A., Morgan J.A. \& Dudareva N. (2017) Plant Volatiles: Going 'In' but not 'Out' of Trichome Cavities. Trends in Plant Science 22, 930-938.

Togashi K., Goto M., Rim H., Hattori S., Ozawa R2. \& Arimura G.I. (2019) Mint companion plants attract the predatory mite Phytoseiulus persimilis .Scientific Report9 , 1704. 
Teoh K.H., Polichuk D.R., Reed D.W., Nowak G. \& Covello P.S. (2006)Artemisia annua L. (Asteraceae) trichome-specific cDNAs reveal CYP71AV1, a cytochrome P450 with a key role in the biosynthesis of the antimalarial sesquiterpene lactone artemisinin. FEBS letters 580, 1411-1416.

Wang Q., Reddy V.A., Panicker D., Mao H.Z., Kumar N., Rajan C., .. Sarojam R. (2016) Metabolic engineering of terpene biosynthesis in plants using a trichome-specific transcription factor $M s$ YABBY5 from spearmint (M. spicata ). Plant Biotechnology Journal 14, 1619-1632.

Wang Z., Ye S., Li J., Zheng B., Bao M. \& Ning G. (2011) Fusion primer and nested integrated PCR (FPNI-PCR): a new high-efficiency strategy for rapid chromosome walking or flanking sequence cloning. BMC Biotechnology 11, 109.

Wu XJ., Xu S., Zhao PZ., Zhang X., Yao X., Sun Y., ... Ye J. (2019) The orthotospovirus nonstructural protein NSs suppresses plant MYC-regulated jasmonate signaling leading to enhanced vector attraction and performance. PLoS Pathogens, 15, e1007897.

Yamaga Y., Nakanishi K., Fukui H. \& Tabata M. (1993) Intracellular localization of $p$-hydroxybenzoate geranyltransferase, a key enzyme involved in shikonin biosynthesis. Phytochemistry32 , 633-636.

Zhu J. \& Park KC. (2005) Methyl salicylate, a soybean aphid-induced plant volatile attractive to the predator Coccinella septempunctata . Journal of Chemical Ecology $\mathbf{3 1}, 8$.

Figure 1 . Phylogenetic tree of candidate gene. The tree was drawn using the Neighbor-Joining method in the MEGA 7 program and were further plotted by web based iTOL (https://itol.embl.de). (a) The candidate TPSs genes were clustered into TPS-b which highlighted by an arrow in red, and genes in white font are all from lavenders (b) The target gene $L a C Y P 71 D 582$ was clustered into CYP 71 clan which was indicated by an arrow in red; CYPs are selected from 5 clans related to terpene metabolism according to Nelson and Werck-Reichhart (2011). The sequences used to construct the tree can be found in table S2 for TPSs and table S3 for CYPs.

Figure 2.Quantitative real-time PCR (qRT-PCR) analysis of LaTPS7, LaTPS8 and LaCYP71D582 expression in glandular trichome from different developmental stage. Each organ containing glandular trichome on flower and glandular trichome on leaf. The highest expression of three genes was at period of bud in glandular trichome on flower. Transcript levels were normalized to $18 \mathrm{~S}$ rRNA genes. Results represent the mean $\pm \mathrm{SE}$ of three repetitions.

Figure 3.Subcellular localization of LaTPS7, LaTPS8 , and LaCYP71D582 inN. benthamiana. Observation under Confocal Fluorescence of cells of tobacco after injection for three days. Both $L a$ TPS7 and $L a$ TPS 8 were associated with chloroplasts, and $L a$ CYP71D582 was located in the endoplasmic reticulum (ER). Auto, chlorophyll autofluorescence; eGFP , enhanced Green Fluorescent Protein channel image; Light, light microscopy image; Merged, merged image between Auto and eGFP . Post processing of pictures were completed by image J (https://imagej.nih.gov/ij).

Figure 4. $L a$ TPS7 and $L a$ TPS8 enzymatic activity in vitro. Products catalyzed by $L a$ TPS7 and $L a$ TPS8 were shown. La TPS7-GPP: 1.a- Pinene, 2. Camphene, 3. Myrcene, 4. Limonene, 5. Terpinolene, 6. Linalool, 7. Terpineol; La TPS7-NPP: 1. a- Pinene, 2. Camphene, 3. Limonene, 4. Terpinolene, 5. Terpineol, 6. Nerolidol; La TPS8-GPP: 1. $\alpha$-Pinene, 2. $\beta$-Pinene, 3. Sylvestrene, 4. Linalool, 5. Fenchol, 6. Geraniol; La TPS8-NPP: 1.a- Pinene, 2. Limonene, 3. Terpinolene, 4. Terpineol, 5. Nerolidol.

Figure 5.Enzyme activity in vivo . (a) Volatiles tested on tobacco leaves transfected with LaTPS7 or LaTPS8 respectively showing that La TPS7 only produced limonene from tobacco and La TPS8 produced a- pinene and sylvestrene. (b) Volatiles test on tobacco leaves co-expressed withLaCYP71D582-LaTPS\%or with LaCYP71D582-LaTPS8. It indicated that limonene can be converted into carveol by La CYP71D582. (c) Limonene was hydroxylated at C6 by $L a$ CYP71D582 to form carveol.

Figure 6. GUS staining showing the expression pattern of LaTPS7 and LaTPS8 . (a) Histochemical stain of Pro-LaTPS7. 1-3, leaves; 4, flower; 5, trichome; 6, silique. The red arrows shows the wound on leaves 
indicating a wound-induced expression pattern of LaTPS7 . (b) Tissue stain of Pro -LaCYP71D582 . 1, leaf; 2 , flower; 3 , trichome; 4 , silique.

Figure 7. Behavioral responses of aphids and ladybugs toward tobacco leaves expressing LaTPS7 and co-expressed LaTPS7 and LaCYP71D582. The percentage of insects choosing wild type leaves (blue bars) versus transfected leaves (green bars) are shown, which indicates that limonene and carveol are able to repel aphids and attract ladybugs. 90 aphids and 60 ladybugs were texted in total. Asterisks indicate a significant difference of choice towards different odor source using $\chi^{2}$ for ladubugs and variance (ANOVA) for aphids $(* * * P<0.05)$.

Figure 8. A summary of the relationship of tritrophic interaction among lavender-aphid-ladybug. Volatiles including limonene, $a$-pinene catalyzed by terpene synthases in bud stage are able to defend plants against attack from aphids. Sequentially, CYP converts the limonene to carveol with stronger attraction to ladybugs. These regulatory networks work together to protect plants against herbivores.
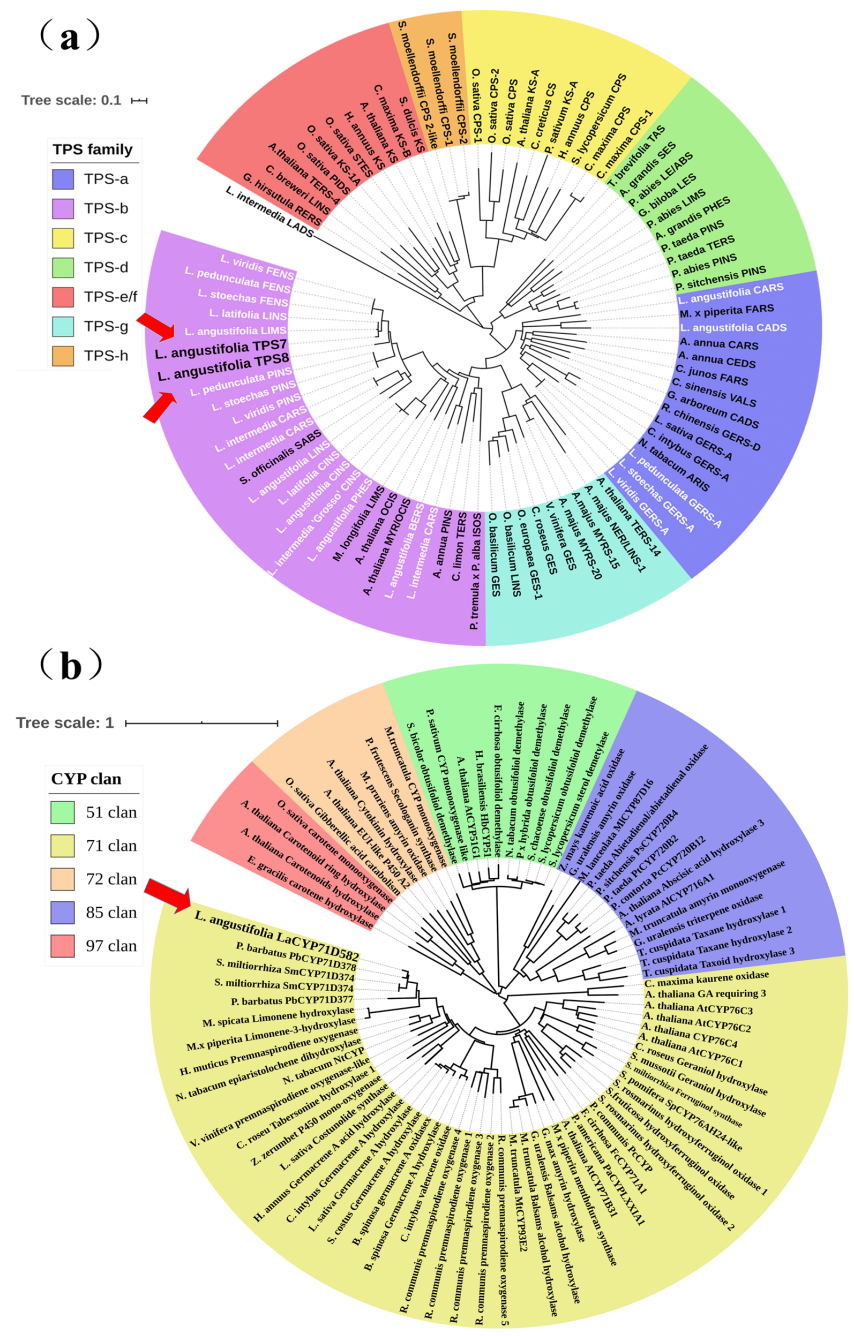


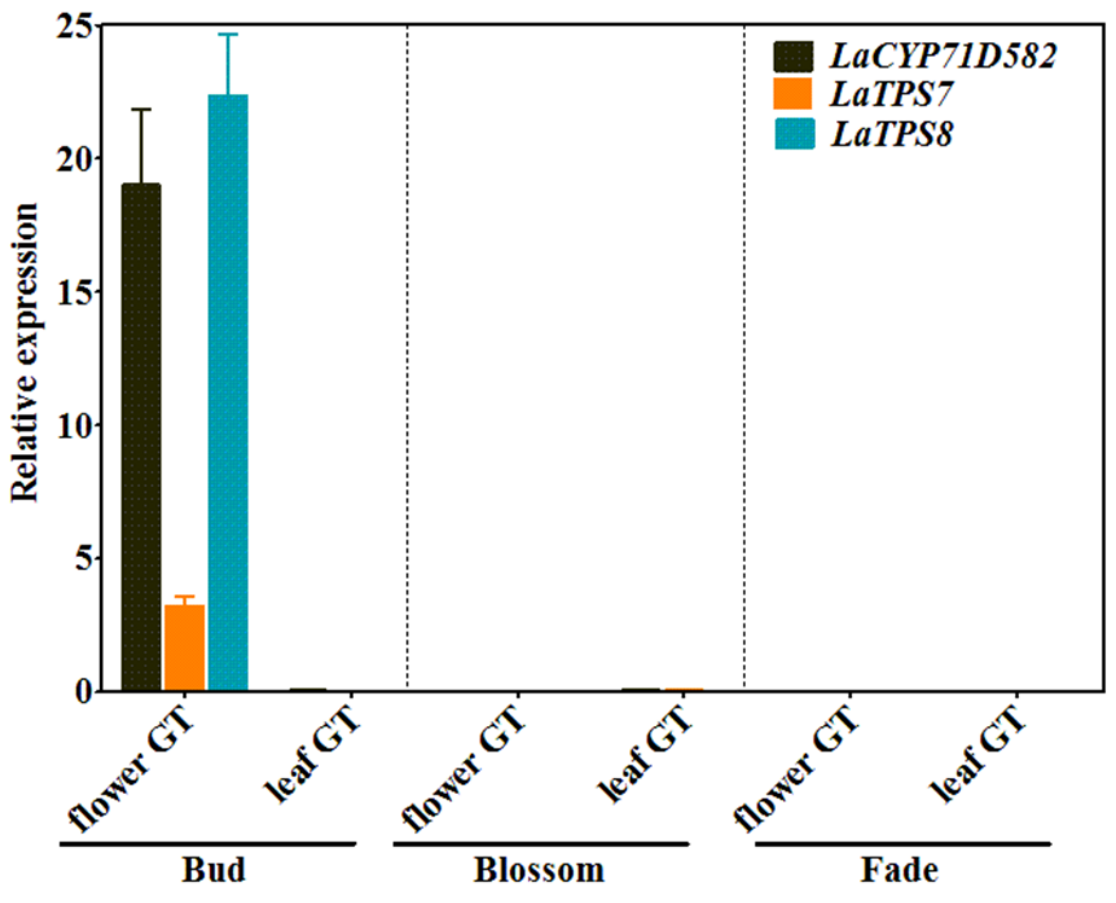




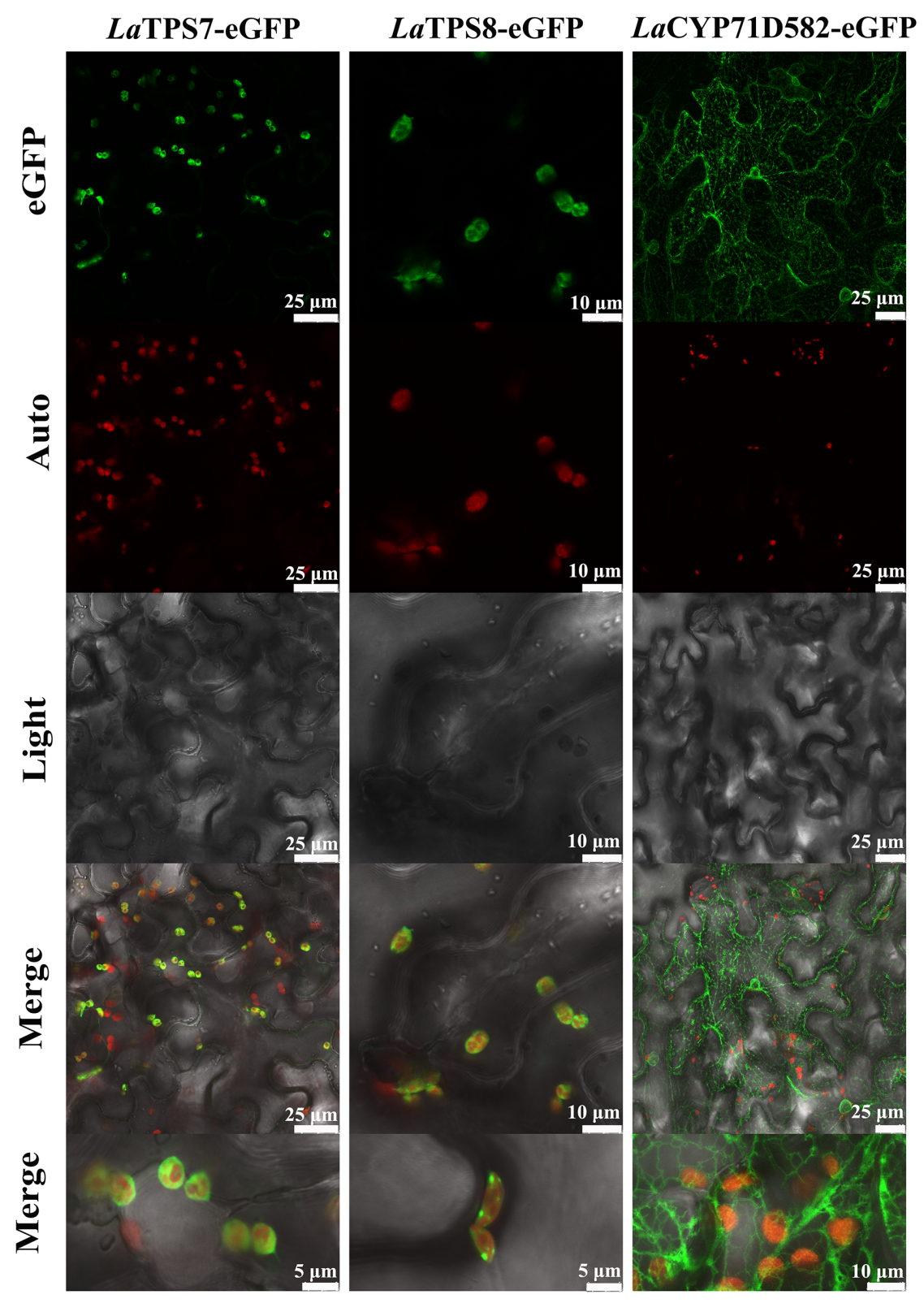



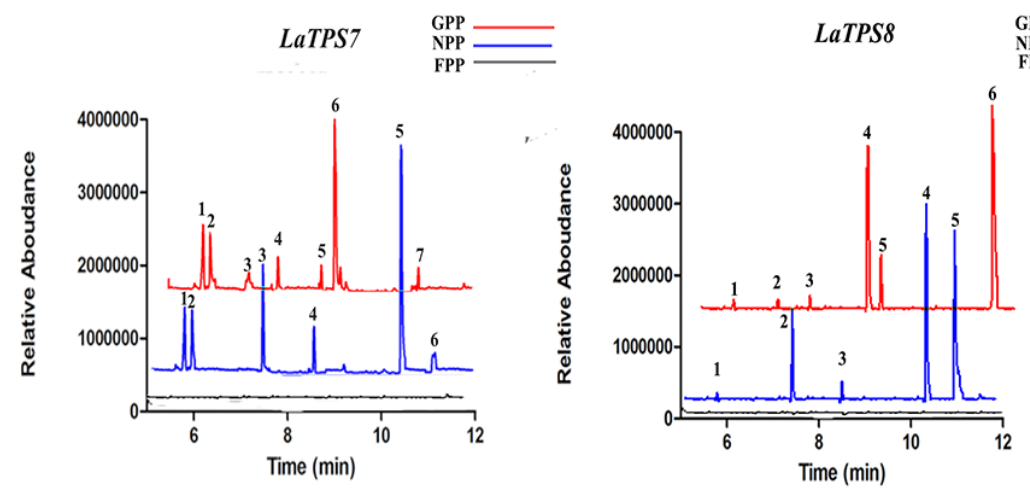

(a)

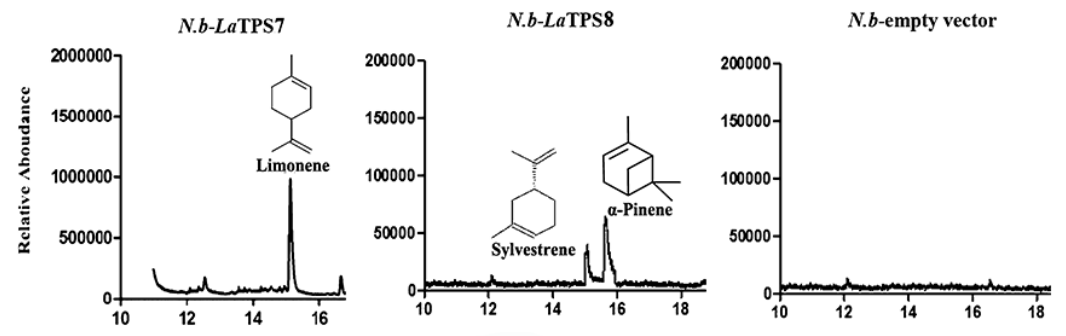

(b)

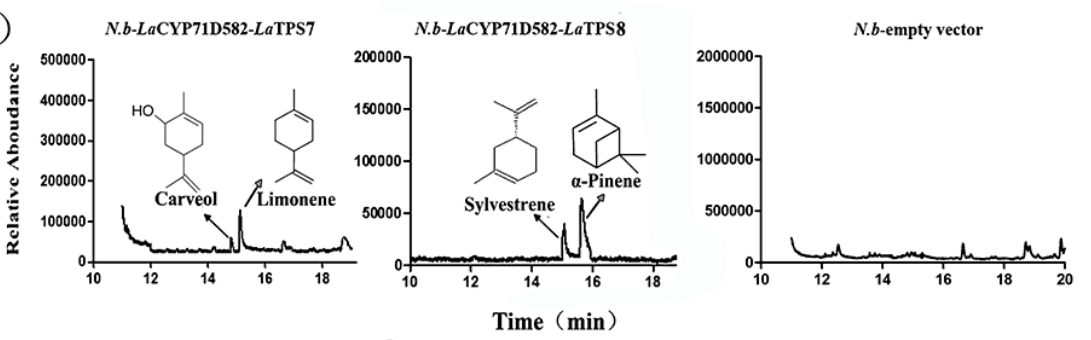

(c)

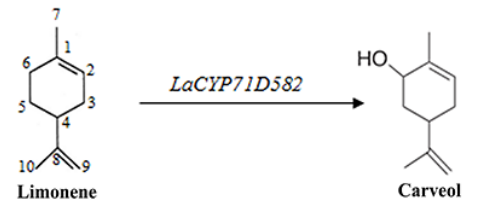


(a)

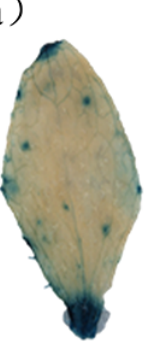

1

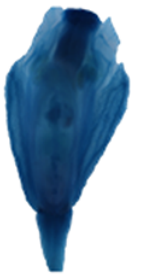

$1.5 \mathrm{~mm}$
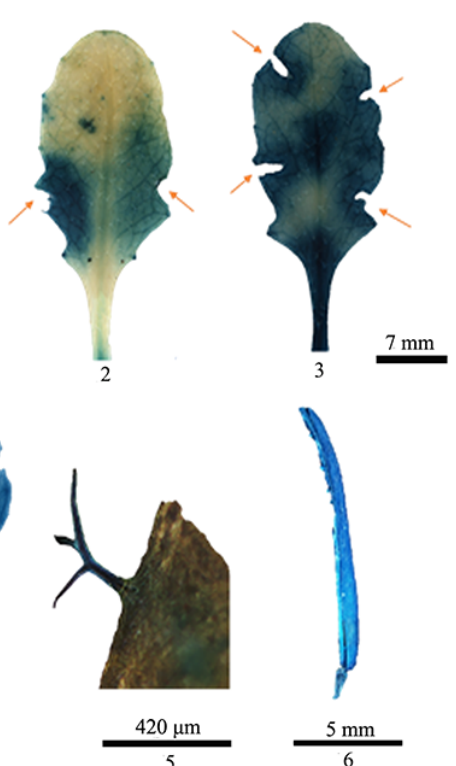

（b）
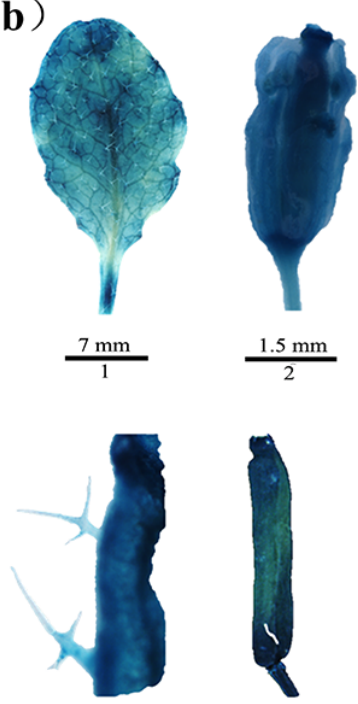

$\frac{420 \mu \mathrm{m}}{3} \frac{5 \mathrm{~mm}}{4}$

(a)

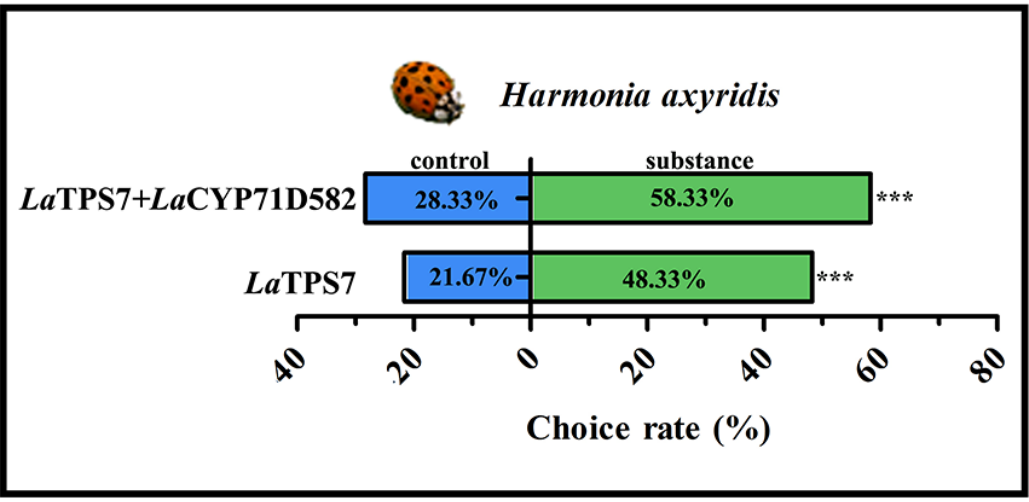

(b)

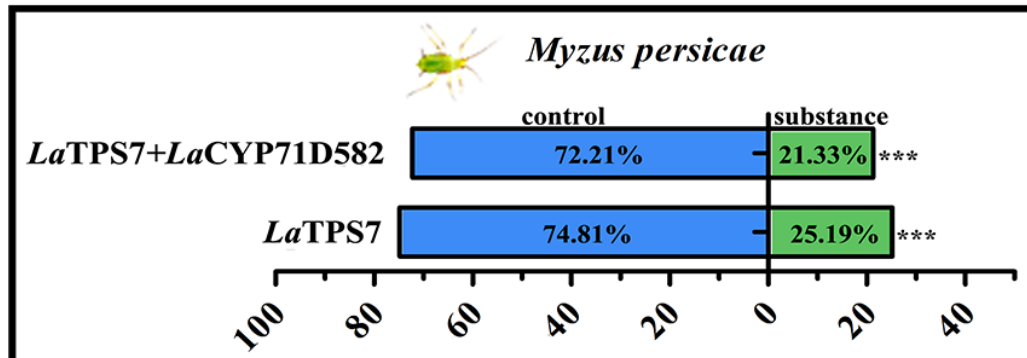

Choice rate (\%) 


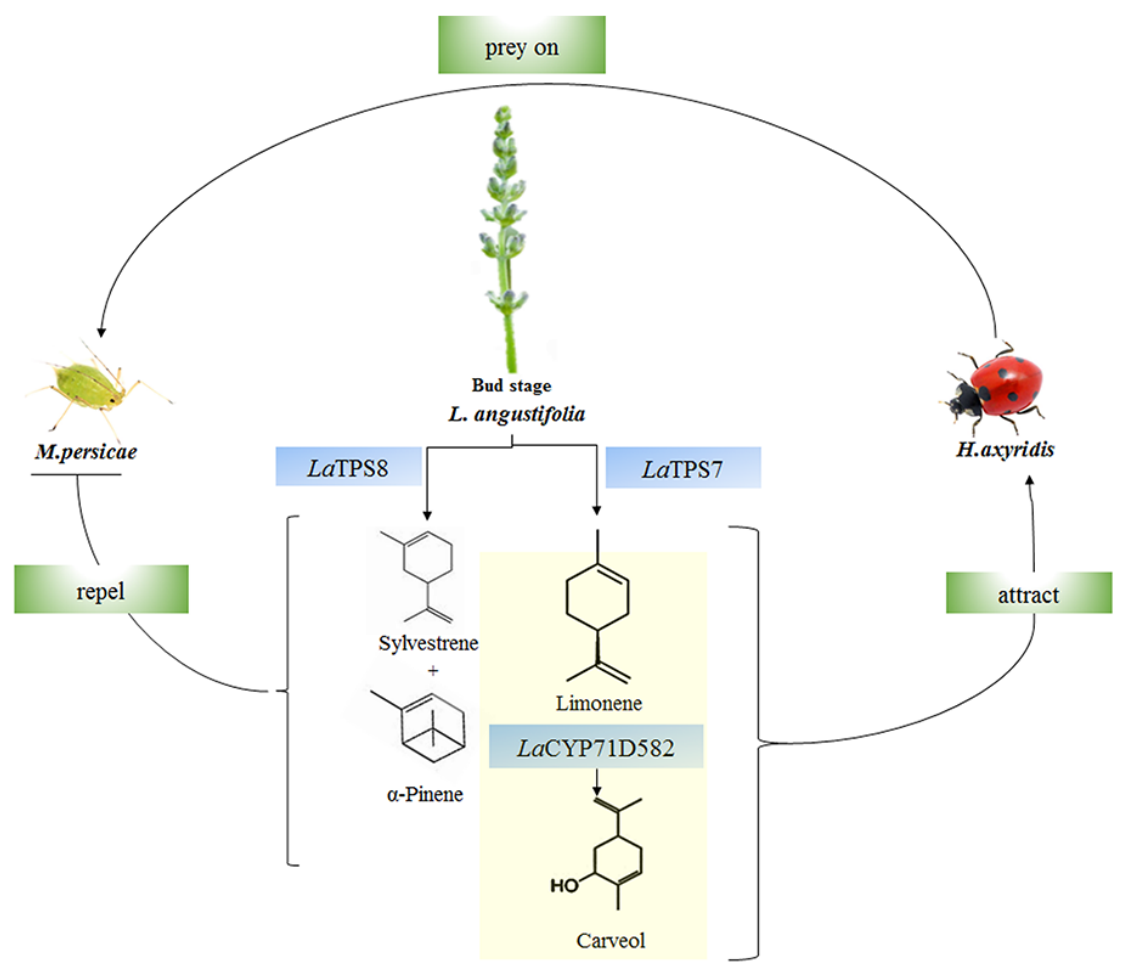

